\title{
SOLUTIONS OF A GOŁA̧B-SCHINZEL-TYPE FUNCTIONAL EQUATION BOUNDED ON 'BIG' SETS IN AN ABSTRACT SENSE
}

\author{
ELIZA JABLOŃSKA
}

(Received 10 June 2009)

\begin{abstract}
It is well known that an exponential real function, which is Lebesgue measurable (Baire measurable, respectively) or bounded on a set of positive Lebesgue measure (of the second category with the Baire property, respectively), is continuous. Here we consider bounded on 'big' set solutions of an equation generalizing the exponential equation as well as the Gołąb-Schinzel equation. Moreover, we unify results into a more general and abstract case.
\end{abstract}

2000 Mathematics subject classification: primary 28E05; secondary 39B52.

Keywords and phrases: Baire category, Lebesgue measurability, Gołąb-Schinzel equation, exponential function, bounded solutions.

\section{Introduction}

Let $\mathbb{N}, \mathbb{R}$ and $\mathbb{C}$ denote the sets of positive integers, real numbers and complex numbers, respectively, and let $\mathbb{K} \in\{\mathbb{R}, \mathbb{C}\}$. Fréchet and Ostrowski proved that if a function $f: \mathbb{R}^{m} \rightarrow \mathbb{R}$ (for $m \in \mathbb{N}$ ) is Lebesgue measurable or bounded on a set of the positive Lebesgue measure and satisfies the exponential functional equation

$$
f(x+y)=f(x) f(y),
$$

then $f$ is continuous (see, for example, [16, Theorems 9.3.1 and 9.4.3]). A topological analog of this fact also holds; more precisely, each solution of (1.1) mapping a linear topological space over $\mathbb{R}$ into $\mathbb{R}$, which is Baire measurable or bounded on a set of the second category with the Baire property, is continuous (see, for example, [16, Theorem 9.3.2]). We also know that these results do not hold in the complex case (see [2, Theorem 2 and Remark]).

Here we characterize solutions of the equation

$$
f(x+M(f(x)) y)=f(x) f(y),
$$

(C) 2010 Australian Mathematical Publishing Association Inc. 0004-9727/2010 \$16.00 
assuming that a function $f$, measurable or bounded on a 'big' set (from an abstract view point), mapping a linear space over $\mathbb{K}$ into $\mathbb{K}$ and a function $M: \mathbb{K} \rightarrow \mathbb{K}$ are unknown. This equation 'connects' Equation (1.1) with the Gołąb-Schinzel equation

$$
f(x+f(x) y)=f(x) f(y) ;
$$

that is, Equations (1.1) and (1.3) are particular cases of (1.2) with $M=1$ and $M=\mathrm{id}_{\mathbb{K}}$, respectively. Equation (1.1) is very well known; for further information we refer the reader to [16, Ch. XIII, Section 1]. The Gołąb-Schinzel equation introduced in [11] is one of the most important composite-type functional equations, because of its applications (and the applications of its generalizations) in the determination of substructures of algebraic structures, in the theory of geometric objects, in the classification of near-rings and quasialgebras, and in differential equations in meteorology and fluid mechanics (for yet another very simple application of (1.3) see Remark 4.4 at the end of this paper). In this connection, equations of Gołąb-Schinzel type have been considered by many authors in various classes of functions (for more information concerning (1.3), its applications and further generalizations we refer to a survey paper [8]).

Lebesgue measurable solutions of an equation of Gołąb-Schinzel type were first studied by Popa [20]. He proved that each Lebesgue measurable real function satisfying (1.3) is continuous or equal to zero almost everywhere. This result confirms the fact that Equations (1.1) and (1.3) have different natures, so looking for solutions of (1.2) seems to be an interesting problem.

Brzdęk [7] proved that each solution $f$ of the equation

$$
f\left(x+f(x)^{n} y\right)=f(x) f(y) \quad \text { with some } n \in \mathbb{N},
$$

mapping a separable $F$-space $X$ over $\mathbb{K}$ into $\mathbb{K}$ with $|f(x)| \subset(0, a)$ for every $x \in A$, for some $a>0$ and for a set $A \subset X$ of the second category with the Baire property, is bounded or continuous. As a consequence, found that every Baire measurable solution of (1.4) is continuous or equal to zero almost everywhere (that is, $f(X \backslash A)=\{0\}$ for a set $A \subset X$ of the first category). An analogous result for solutions $f: \mathbb{R}^{m} \rightarrow \mathbb{R}$ (with $m \in \mathbb{N}$ ) of (1.4) bounded on a set of positive Lebesgue measure has been proved in [13].

Mureńko [18] determined solutions of the following generalization of (1.2):

$$
f(x+M(f(x) y)=f(x) \circ f(y),
$$

where $M: \mathbb{R} \rightarrow \mathbb{R}$, the Lebesgue measurable function $f: \mathbb{R} \rightarrow \mathbb{R}$ and $\circ: \mathbb{R}^{2} \rightarrow \mathbb{R}$ are unknown functions.

In this paper, we generalize the results mentioned above by $[7,13,20]$ for the case of Equation (1.2). We also unify these results using an abstract property of measurability with respect to some families of sets satisfying a certain hypothesis.

Since Sierpiński [22] noticed that there are some similarities between measure and category (see also [19]), some authors have introduced abstract properties of 
measurability, using selected analogies between measure and category, to unify the results concerning those two fields (see, for example, [3, 9, 10, 17, 21]). Our hypothesis refers to the analogy between a generalization of Steinhaus's theorem (see [4]) and its topological analog, that is, a generalization of Piccard's theorem (see, for example, [15]).

Throughout this paper, we denote

$$
A:=f^{-1}(\{1\}), \quad W:=f(X) \backslash\{0\}, \quad F:=\{x \in X: f(x) \neq 0\}
$$

for a function $f: X \rightarrow \mathbb{K}$ mapping a linear space $X$ over $\mathbb{K}$ into $\mathbb{K}$.

\section{Preliminary lemmas}

First, we give some lemmas which will be useful in what follows.

Lemma $2.1[5$, Lemmas 2, 3]. Let $\mathbb{K} \in\{\mathbb{R}, \mathbb{C}\}$, let $B$ be a subgroup of $(X,+)$ and let $V$ be an infinite subgroup of $(\mathbb{K} \backslash\{0\}, \cdot)$ such that $V B \subset B$. Moreover, in the case when $\mathbb{K}=\mathbb{C}$ assume that $V \backslash \mathbb{R} \neq \emptyset$. Then, for every $x \in B$, the set $B_{x}=\{a \in \mathbb{K}: a x \in B\}$ is dense in $\mathbb{K}$ (with the usual topology in $\mathbb{K}$ ).

In the next lemma we collect together some basic properties of the functions satisfying (1.2), which have been proved in [12] (see Lemmas 2 and 3 and Propositions 1 and 2).

Lemma 2.2. Let $X$ be a linear space over $\mathbb{K} \in\{\mathbb{R}, \mathbb{C}\}, f: X \rightarrow \mathbb{K}, f \neq 0, f \neq 1$ (that is, $f$ is neither the constant function 0 nor the constant function 1) and $M: \mathbb{K} \rightarrow \mathbb{K}$. If $f$ and $M$ satisfy (1.2), then the following properties hold:

(i) $f\left(M(f(x))^{-1}(z-x)\right)=f(z) f(x)^{-1}$ for $x \in F$ and $z \in X$;

(ii) $(M \circ f)^{-1}(\{0\})=f^{-1}(\{0\})$;

(iii) $M(a) A=A$ for $a \in W$;

(iv) $A$ is a subgroup of $(X,+)$;

(v) $A \backslash\{0\}$ is the set of periods of $f$;

(vi) $W$ is a subgroup of $(\mathbb{K} \backslash\{0\}, \cdot)$;

(vii) $y-x \in A$ for every $x, y \in F$ where $f(x)=f(y)$;

(viii) there exists a function $w: W \rightarrow X$ such that $F=w(W)+A$;

(ix) if, moreover, $M(1)=1$ and $M \circ f \neq 1$, then $0 \in f(X)$.

LEMma 2.3 (See [14, Lemma 3]). Let $X$ be a linear space over $\mathbb{K} \in\{\mathbb{R}, \mathbb{C}\}, f: X \rightarrow$ $\mathbb{K}, M: \mathbb{K} \rightarrow \mathbb{K}, f \neq 0, M(1)=1$ and $M(W) \backslash\{1\} \neq \emptyset$. If $f$ and $M$ satisfy (1.2), then there exists an $x_{0} \in X$ such that

$$
F \subset(M(W)-1) x_{0}+\operatorname{lin}_{\mathbb{K}_{0}} A,
$$

where $\operatorname{lin}_{\mathbb{K}_{0}}$ A denotes the linear subspace of $X$ spanned by A over the field

$$
\mathbb{K}_{0}= \begin{cases}\mathbb{R} & \text { if } M(W) \subset \mathbb{R}, \\ \mathbb{C} & \text { if } M(W) \backslash \mathbb{R} \neq \emptyset .\end{cases}
$$


Furthermore, if $A=\operatorname{lin}_{\mathbb{K}_{0}} A$, then $x_{0} \notin A$,

$$
f(x)= \begin{cases}a & \text { for } x \in(M(a)-1) x_{0}+A \text { and } a \in W, \\ 0 & \text { otherwise }\end{cases}
$$

and $\left.M\right|_{f(X)}$ is injective and multiplicative.

The proof of this lemma runs in the same way as in the case when $\mathbb{K}=\mathbb{R}$, so we refer the reader to the proof of Lemma 3 in [14].

\section{Ideals and a hypothesis}

Let $X$ be a linear space over a field $\mathbb{K}$. First, let us recall that a family $\mathcal{I} \subset 2^{X}$ is called a proper linearly invariant $\sigma$-ideal provided:

(1) $\mathcal{I} \neq\{\emptyset\}$ and $\mathcal{I} \neq 2^{X}$

(2) $2^{A} \subset \mathcal{I}$ for each $A \in \mathcal{I}$;

(3) $\bigcup_{n \in \mathbb{N}} A_{n} \in \mathcal{I}$ for every $\left\{A_{n}\right\}_{n \in \mathbb{N}} \subset \mathcal{I}$;

(4) $x+A \in \mathcal{I}$ and $a A \in \mathcal{I}$ for every $x \in X, a \in \mathbb{K}$ and $A \in \mathcal{I}$.

In further considerations we use the following hypothesis.

(H) $\quad X$ is a linear topological space over $\mathbb{K} \in\{\mathbb{R}, \mathbb{C}\}, \mathfrak{M}$ is a $\sigma$-algebra of its subsets and there exists a nontrivial proper linearly invariant $\sigma$-ideal $\mathcal{I} \subset 2^{X}$ such that:

(H1) $\operatorname{int}(A+B) \neq \emptyset$ for every $A \in \mathfrak{M} \backslash \mathcal{I}$ and $B \in 2^{X} \backslash \mathcal{I}$,

(H2) $\operatorname{int}[(g(A)+1) \cdot(g(A)+1)] \neq \emptyset$ for every $A \in \mathfrak{M} \backslash \mathcal{I}$ and $g \in X^{*} \backslash\{0\}$,

where $X^{*}$ is the space of all linear continuous functionals on $X$.

The above hypothesis (especially condition (H1)) corresponds to the hypothesis (M) introduced in [9] and the hypothesis (H) from [3].

Example 3.1. Let $X$ be an $F$-space over $\mathbb{K} \in\{\mathbb{R}, \mathbb{C}\}$. Let $\mathfrak{M}$ denote the $\sigma$-algebra of all subsets of $X$ having the Baire property and let $\mathcal{I}$ denote the $\sigma$-ideal of all subsets of $X$ of the first category. By [7, Lemma 5], for every $A \subset X$ of the second category with the Baire property (in $X$ ) and $g \in X^{*} \backslash\{0\}$, the set $g(A)$ is of the second category with the Baire property (in $\mathbb{K}$ ). Hence, in view of a generalization of Piccard's theorem (see, for example, [15, Theorem 2]), the hypothesis (H) holds.

Example 3.2. Let $X=\mathbb{K}^{n}$, where $\mathbb{K} \in\{\mathbb{R}, \mathbb{C}\}$ and $n \in \mathbb{N}$. Let $\mathfrak{M}$ denote the $\sigma$-algebra of all Lebesgue measurable subsets of $\mathbb{K}^{n}$ and let $\mathcal{I}$ denote the $\sigma$-ideal of all Lebesgue zero subsets of the space. In view of a generalization of Steinhaus's theorem (see [4, Theorem 1]), condition (H1) holds. Condition (H2) holds as a consequence of Steinhaus's theorem (see [6, Lemma 10]) and the following fact.

Let $g: \mathbb{K}^{n} \rightarrow \mathbb{K}$ be a nontrivial linear functional and $V \subset \mathbb{K}$, where $\mathbb{K} \in\{\mathbb{R}, \mathbb{C}\}$. If $D$ is a set of positive inner Lebesgue measure in $\mathbb{K}^{n}$, then $g(D)$ has positive inner Lebesgue measure in $\mathbb{K}$. 
The proof of this fact is exactly the same as in the case when $\mathbb{K}=\mathbb{R}$, so we refer the reader to the proof of Lemma 6 in [13].

Taking $\mathcal{E}:=\left\{z \in \mathbb{K}: \exists_{k \in \mathbb{N}} z^{k}=1\right\}$ we prove a lemma that will be useful in what follows.

Lemma 3.3. Assume (H). If $f: X \rightarrow \mathbb{K}$ and $M: \mathbb{K} \rightarrow \mathbb{K}$ satisfy (1.2), $M(1)=1$, $M \circ f \neq 1$ and there is a $D \in \mathfrak{M} \backslash \mathcal{I}$ such that $D \subset F$, then card $f(X)>\aleph_{0}$ and $M(W) \backslash \mathcal{E} \neq \emptyset$.

PROOF. For an indirect proof, suppose that

$$
\text { for every } b \in W \text { there exists a } k \in \mathbb{N} \text { such that }(M(b))^{k}=1 \text {. }
$$

If $M(W)=\{1\}$, then $f(x+y)=f(x) f(y) \neq 0$ for every $x, y \in F$ and thus $F+F \subset F$. By $(\mathrm{H} 1)$

$$
\emptyset \neq \operatorname{int}(D+D) \subset \operatorname{int}(F+F) \subset \operatorname{int} F .
$$

Hence, according to Lemma 2.2(i), $0 \in$ int $F$. Then, for each $x \in X$, there exists an $n \in \mathbb{N}$ such that $(1 / n) x \in F$. This implies $x=n(1 / n) x \in n F \subset F$. In this way, we obtain $X=F$, which contradicts Lemma 2.2(ix). Thus $M(W) \backslash\{1\} \neq \emptyset$.

Using (1.2) we can prove by induction that, for every $n \in \mathbb{N} \backslash\{1\}$ and $x \in X$,

$$
(f(x))^{n}=f\left(x\left(1+\sum_{k=1}^{n-1}(M(f(x)))^{k}\right)\right),
$$

and hence, for each $x \in X$ with $M(f(x)) \neq 1$,

$$
(f(x))^{n}=f\left(\frac{1-(M(f(x)))^{n}}{1-M(f(x))} \cdot x\right) .
$$

By Lemma 2.2(iv), $f(0)=1$. Thus, in view of (3.1) and (3.3), we find that for each $b \in W$ with $M(b) \neq 1$ there exists $k \in \mathbb{N}$ fulfilling $b^{k}=1$. Hence $\{a \in W: M(a) \neq$ 1) $\subset \mathcal{E}$.

Now we show that $\{a \in W: M(a)=1\} \subset \mathcal{E}$. Fix $x \in X$ such that $M(f(x))=1$. Since $M(W) \backslash\{1\} \neq \emptyset$, there exists a $y \in X$ with $M(f(y))^{k}=1$ for some $k \geq 2$. Then, by Lemma 2.2(ii), $f(x) f(y) \neq 0$. In view of (1.2),

$$
f(y+M(f(y)) x)=f(x) f(y)=f(x+M(f(x)) y)=f(x+y) .
$$

Hence, according to Lemma 2.2(vii), we find that

$$
x-M(f(y)) x \in A .
$$

Now we prove by induction that

$$
x-M(f(y))^{n} x \in A \quad \text { for each } n \in\{1,2, \ldots, k-1\} .
$$


For $n=1$, (3.5) coincides with (3.4). Assume that

$$
x-M(f(y))^{n} x \in A \text { for some } n \in\{2, \ldots, k-2\} .
$$

Using Lemma 2.2(iii),

$$
M(f(y)) x-M(f(y))^{n+1} x \in M(f(y)) A=A .
$$

Now, in view of (3.4) and Lemma 2.2(iv),

$$
x-M(f(y))^{n+1} x \in A+A=A .
$$

This ends the proof of condition (3.5).

By (3.5) and Lemma 2.2(iv), we obtain

$$
A \ni \sum_{n=1}^{k-1}\left(x-M(f(y))^{n} x\right)=k x-\sum_{n=0}^{k-1} M(f(y))^{n} x=k x-\frac{1-M(f(y))^{k}}{1-M(f(y))} \cdot x .
$$

Since $M(f(y))^{k}=1$, we have that $k x \in A$. But $M(f(x))=1$. Thus, in view of (3.2), $1=f(k x)=f(x)^{k}$. Hence, by Lemma 2.2(ii), for each $b \in W$ such that $M(b)=1$, there exists a $k \in \mathbb{N}$ fulfilling $b^{k}=1$. This means that $\{a \in W: M(a)=1\} \subset \mathcal{E}$.

In this way, we find that $W \subset \mathcal{E}$ and, consequently, card $W \leq \aleph_{0}$. By Lemma 2.2(viii), there exists a function $w: W \rightarrow X$ such that

$$
F=\bigcup_{a \in W}(w(a)+A) \text {. }
$$

Since $D \notin \mathcal{I}$, we have that $F \notin \mathcal{I}$ and, consequently, $A \notin \mathcal{I}$. Furthermore, using (H1) and Lemma 2.2(v),

$$
\emptyset \neq \operatorname{int}(D+A) \subset \operatorname{int}(F+A) \subset \operatorname{int} F .
$$

Hence $A$ is of the second category. In view of Piccard's theorem, this implies $0 \in \operatorname{int}(\mathrm{cl} A-\operatorname{cl} A)$ and consequently, by Lemma $2.2(\mathrm{iv}), \operatorname{int}(\operatorname{cl} A)=X$. Hence $A$ is dense in $X$ and, according to Lemma 2.2(v), $X=F+A=F$, which contradicts Lemma 2.2(ix).

\section{Main results}

In what follows, for a set $T \subset \mathbb{K}$ we put $|T|:=\{|a|: a \in T\}$ and $S:=\{z \in \mathbb{C}$ : $|z|=1\}$. Now we prove our main theorem.

Theorem 4.1. Assume (H). Let $D \in \mathfrak{M} \backslash \mathcal{I}, f: X \rightarrow \mathbb{K}$ and

$$
|f(D)| \subset(0, a) \text { for some } a>0,
$$

and let $M: \mathbb{K} \rightarrow \mathbb{K}$ be continuous. Let, moreover, $\operatorname{int}(S \backslash f(D)) \neq \emptyset$ (with respect to the usual topology in $S$ ) in the case when $W \subset S$. Functions $f$ and $M$ satisfy (1.2) if and only if one of the following conditions holds: 
(i) $f=1$;

(ii) $M \circ f=1$ and $f: X \rightarrow \mathbb{K} \backslash\{0\}$ is an exponential function;

(iii) there exist some $c>0$ and a nontrivial continuous $\mathbb{R}$-linear functional $g: X \rightarrow \mathbb{R}$ such that either

$$
\begin{gathered}
f(x)=|g(x)+1|^{1 / c} \operatorname{sgn}(g(x)+1) \quad \text { for } x \in X, \\
M(y)=|y|^{c} \operatorname{sgn} y \quad \text { for } y \in \mathbb{R}
\end{gathered}
$$

or

$$
\begin{gathered}
f(x)=(\max \{0, g(x)+1\})^{1 / c} \quad \text { for } x \in X, \\
M(y)=y^{c} \quad \text { for } y \in[0, \infty) ;
\end{gathered}
$$

(iv) there exist a continuous multiplicative bijection $H: \mathbb{C} \rightarrow \mathbb{C}$ and a nontrivial continuous $\mathbb{C}$-linear functional $g: X \rightarrow \mathbb{C}$ such that

$$
\begin{gathered}
f(x)=H^{-1}(g(x)+1) \quad \text { for } x \in X, \\
M(y)=H(y) \quad \text { for } y \in \mathbb{C} .
\end{gathered}
$$

REMARK 4.2. Clearly, there are two constant solutions of (1.2), $f=0$ and $f=1$, but in the above theorem condition (4.1) implies $f \neq 0$.

Proof of Theorem 4.1. First, assume that functions $f$ and $M$ satisfy (1.2). The case $f=1$ is clear, so assume that $f \neq 1$. Define a function $\widetilde{M}: \mathbb{K} \rightarrow \mathbb{K}$ as

$$
\tilde{M}(a)=\frac{M(a)}{M(1)} \quad \text { for each } a \in \mathbb{K}
$$

and put $x=0$ in (1.2). Then, in view of Lemma 2.2(iv), we obtain $f(M(1) y)=$ $f(y) f(0)=f(y)$ for each $y \in X$. By Lemma 2.2(ii), we have $M(1) \neq 0$. Hence, replacing $y$ by $z / M(1), f(z / M(1))=f(z)$ for $z \in X$. Consequently, for every $x, z \in X$,

$$
f(x+\tilde{M}(f(x)) z)=f\left(x+M(f(x)) \frac{z}{M(1)}\right)=f(x) f\left(\frac{z}{M(1)}\right)=f(x) f(z) .
$$

This means that the functions $f$ and $\tilde{M}$ given by (4.2) also satisfy (1.2), where $\widetilde{M}(1)=1$. If $M \circ f=c$, then $\widetilde{M} \circ f=1$. Hence, using (1.2), $f$ is an exponential function. Since $f \neq 0$, we have that $0 \notin f(X)$ (if $f\left(x_{0}\right)=0$ for an $x_{0} \in X$, then $f(x)=f\left(x-x_{0}\right) f\left(x_{0}\right)=0$ for each $\left.x \in X\right)$. Putting $x=0$ in (1.2), we have that $f((c-1) y)=1$ for each $y \in X$. Thus $c=1$ (because $f \neq 1$ ).

Now suppose that $M \circ f \neq c$. Then $\tilde{M} \circ f \neq 1$ and, in view of Lemma 2.2(ix), $0 \in f(X)$. Since $f \neq 1$, by Lemma 2.2(ii), $\tilde{M}(0)=0$. First we show that $\operatorname{lin}_{\mathbb{K}_{0}} A \neq X$ for the field $\mathbb{K}_{0}$ given by (2.2). So suppose that this is not the case. Then $A$ must contain a basis for $X$. Moreover, by Lemma 2.2(iii),

$$
a A=A \quad \text { for } a \in W_{0},
$$


where $W_{0}$ is a multiplicative group generated by $\tilde{M}(W)$. Using Lemma 3.3, we find that $W_{0}$ is an infinite subgroup of the group $\left(\mathbb{K}_{0} \backslash\{0\}, \cdot\right)$. Consequently, in view of Lemma 2.1, $A$ is dense in $X$. Define a set

$$
D_{0}:= \begin{cases}\{x \in F:|f(x)|<a\} & \text { if } W \backslash S \neq \emptyset, \\ \{x \in F: f(x) \notin \operatorname{int}(S \backslash f(D))\} & \text { if } W \subset S .\end{cases}
$$

Clearly $D \subset D_{0}$. Moreover, by Lemma 2.2(v), $A+D_{0} \subset D_{0}$. Hence we obtain

$$
A \cap\left[\left(X \backslash D_{0}\right)-D_{0}\right]=\emptyset .
$$

Thus $X \backslash D_{0} \in \mathcal{I}$, because otherwise, in view of (H1), we would have

$$
\emptyset \neq \operatorname{int}\left(\left(X \backslash D_{0}\right)-D\right) \subset \operatorname{int}\left(\left(X \backslash D_{0}\right)-D_{0}\right),
$$

which contradicts the density of $A$. Now, according to Lemma 2.2(vi), there is a sequence $\left(x_{m}\right)_{m \in \mathbb{N}} \subset F$ such that, for each $x \in F$, there exists an $m \in \mathbb{N}$ with

$$
f\left(x_{m}+\tilde{M}\left(f\left(x_{m}\right)\right) x\right)=f\left(x_{m}\right) f(x) \in f\left(X \backslash D_{0}\right)
$$

(in the case when $W \subset S$, in view of Lemmas 2.2(vi) and 3.3, $W$ is dense in $S$ and hence $\left(x_{m}\right)_{m \in \mathbb{N}}$ is a countable subset of $F$ such that $\left(f\left(x_{m}\right)\right)_{m \in \mathbb{N}}$ is dense in $S$ ). According to Lemma 2.2(ii),

$$
D \subset F \subset \bigcup_{m \in \mathbb{N}}\left(\left(X \backslash D_{0}\right)-x_{m}\right) \tilde{M}\left(f\left(x_{m}\right)\right)^{-1} .
$$

Then $X \backslash D_{0} \in \mathcal{I}$ implies $D \in \mathcal{I}$, which contradicts the assumption. In this way, we obtain $\operatorname{lin}_{\mathbb{K}_{0}} A \neq X$.

By Lemma 3.3, $\tilde{M}(W) \backslash\{1\} \neq \emptyset$. Thus, in view of Lemma 2.3, there exists an $x_{0} \in X$ such that (2.1) holds. Hence

$$
F-F \subset(\tilde{M}(W)-\tilde{M}(W)) x_{0}+\operatorname{lin}_{\mathbb{K}_{0}} A .
$$

Next, by $(\mathrm{H} 1), \emptyset \neq \operatorname{int}(D-D) \subset \operatorname{int}(F-F)$. Since $\operatorname{lin}_{\mathbb{K}_{0}} A \neq X$, we have that $x_{0} \notin \operatorname{lin}_{\mathbb{K}_{0}} A$. Consequently,

$$
\operatorname{int}_{\mathbb{K}_{0}}(\tilde{M}(W)-\tilde{M}(W)) \neq \emptyset
$$

and

$$
\mathbb{K}_{0} x_{0}+\operatorname{lin}_{\mathbb{K}_{0}} A=X
$$

Thus

$$
\operatorname{int}_{\mathbb{K}_{0}}\left(W_{0}-W_{0}\right) \neq \emptyset .
$$

Moreover, by (4.3) and Lemma 2.2(iv), $\mathbb{K}_{0} A \subset A$, which implies $A=\operatorname{lin}_{\mathbb{K}_{0}} A$. Then, according to Lemma 2.3,

$$
F=(\tilde{M}(W)-1) x_{0}+A
$$


and $\left.\tilde{M}\right|_{f(X)}$ is injective and multiplicative. Moreover, from (4.4) we find that $A$ is a hyperplane of $X$ (that is, $\operatorname{codim}_{\mathbb{K}_{0}} A=1$ ). So, we can define a linear functional $g: X \rightarrow \mathbb{K}_{0}$ as follows:

$$
g\left(a x_{0}+y\right)=a \quad \text { for every } a \in \mathbb{K}_{0} \text { and } y \in A .
$$

In view of (4.5)

$$
g(x)=\tilde{M}(f(x))-1 \quad \text { for each } x \in F .
$$

Hence $g(D)+1 \subset \tilde{M}(W)$ and $g$ is bounded on $D$. Consequently, by (H1), $g$ is bounded on $\operatorname{int}(D+D) \neq \varnothing$ and thus $g$ is continuous. Then, using (H2) and the continuity of $\widetilde{M}$,

$$
\operatorname{int}_{\mathbb{K}_{1}} \tilde{M}^{-1}((g(D)+1)(g(D)+1)) \neq \emptyset
$$

for the field

$$
\mathbb{K}_{1}= \begin{cases}\mathbb{R} & \text { if } W \subset \mathbb{R}, \\ \mathbb{C} & \text { if } W \backslash \mathbb{R} \neq \emptyset .\end{cases}
$$

Next, by (H2), Lemma 2.2(vi) and the multiplicativity of $\left.\widetilde{M}\right|_{f(X)}$,

$$
\begin{gathered}
\operatorname{int}_{\mathbb{K}_{0}} \tilde{M}(W) \supset \operatorname{int}_{\mathbb{K}_{0}}(\tilde{M}(W) \cdot \tilde{M}(W)) \supset \operatorname{int}_{\mathbb{K}_{0}}((g(D)+1) \cdot(g(D)+1)) \neq \emptyset, \\
\operatorname{int}_{\mathbb{K}_{1}} W \supset \operatorname{int}_{\mathbb{K}_{1}}(W \cdot W) \supset \operatorname{int}_{\mathbb{K}_{1}}\left(\tilde{M}^{-1}(g(D)+1) \cdot \tilde{M}^{-1}(g(D)+1)\right) \\
=\operatorname{int}_{\mathbb{K}_{1}} \tilde{M}^{-1}((g(D)+1) \cdot(g(D)+1)) \neq \emptyset,
\end{gathered}
$$

and, consequently, $W, \widetilde{M}(W) \in\{\mathbb{C} \backslash\{0\}, \mathbb{R} \backslash\{0\},(0, \infty)\}$.

In the case when $\tilde{M}(W)=(0,+\infty)$, in view of (4.4) and (4.5), $X \backslash F=$ $(-\infty,-1] x_{0}+A$. Hence, by (4.6), $g(x) \in(-\infty,-1]$ for $x \notin F$. Moreover, by Lemma 2.2(ii), $\widetilde{M}(f(x))=0$ for $x \notin F$ so, in view of (4.7),

$$
\tilde{M}(f(x))=\max \{0, g(x)+1\} \quad \text { for } x \in X .
$$

Finitely, consider the case when $\tilde{M}(W) \in\{\mathbb{R} \backslash\{0\}, \mathbb{C} \backslash\{0\}\}$. Then, by (4.4) and (4.5), $X \backslash F=-x_{0}+A$. Hence, in view of (4.6), $g(x)=-1$ for $x \notin F$. On the other hand, using Lemma 2.2(ii), $\widetilde{M}(f(x))=0$ for $x \notin F$. Thus, according to (4.7),

$$
\tilde{M}(f(x))=g(x)+1 \quad \text { for } x \in X .
$$

So, $\left.\tilde{M}\right|_{f(X)}$ is injective and multiplicative and $\tilde{M} \circ f$ is given by (4.8) or (4.9). Hence, by (4.2), $\left.M\right|_{f(X)}$ is injective,

$$
M(1) M(a b)=M(a) M(b) \quad \text { for } a, b \in f(X)
$$

and either

$$
M(f(x))=M(1)(g(x)+1) \quad \text { for } x \in X
$$

or

$$
M(f(x))=M(1) \max \{0, g(x)+1\} \quad \text { for } x \in X .
$$


Moreover, by Lemma 2.2(ii), $F=\{x \in X: g(x)>-1\}$ when $M \circ f$ is given by (4.11), and $F=\{x \in X: g(x) \neq-1\}$ in the other case. From (4.10) we find that, for every $x, y \in F$,

$$
M(f(x) f(y))=\frac{M(f(x)) M(f(y))}{M(1)}=(g(x)+1)(g(y)+1) M(1) .
$$

On the other hand, in view of (1.2) and Lemma 2.2(ii), $M(f(x+M(f(x)) y)) \neq 0$ for $x, y \in F$ and hence

$$
\begin{aligned}
M(f(x+M(f(x)) y)) & =M(1)(g(x+M(f(x)) y)+1) \\
& =M(1)(g(x)+g(M(f(x)) y)+1) \\
& =M(1)\left(g(x)+1+\frac{M(f(x))}{M(1)} g(M(1) y)\right) \\
& =M(1)(g(x)+1)(g(M(1) y)+1) .
\end{aligned}
$$

Now, from (1.2), we obtain $g(y)=g(M(1) y)$ for each $y \in F$. Thus

$$
g((1-M(1)) y)=0 \quad \text { for } y \in F .
$$

Suppose that $M(1) \neq 1$. Since $M \circ f \neq$ const, there exists a $z \in X$ such that $g(z) \neq 0$. Let $w=(1-M(1))^{-1} z$. Then, in view of (4.13), $w \notin F$. Hence $g(w) \leq-1$, when $M \circ f$ is given by (4.11), and $g(w)=-1$ in the other case. Now, by $\mathbb{R}$-homogeneity of $g$, there exists an $r \in \mathbb{R} \backslash\{0\}$ such that $r w \in F$. This means that

$$
0=g((1-M(1)) r w)=g(r z)=r g(z) \neq 0 .
$$

This contradiction proves that $M(1)=1$. Thus, from (4.2), $\widetilde{M}=M$.

Hence, by Lemma 2.2(ix), $f(X), M(f(X)) \in\{\mathbb{C}, \mathbb{R},[0, \infty)\}$. Moreover, the multiplicative bijections $M: \mathbb{C} \rightarrow \mathbb{R}, M: \mathbb{C} \rightarrow[0, \infty), M: \mathbb{R} \rightarrow[0, \infty), M: \mathbb{R} \rightarrow \mathbb{C}$, $M:[0, \infty) \rightarrow \mathbb{C}$ and $M:[0, \infty) \rightarrow \mathbb{R}$ do not exist (since $\tilde{M}(a)=-1$ if and only if $a=-1$ and $M(a) \in\{i,-i\}$ if and only if $a \in\{-i, i\})$. Hence $f(X)=M(f(X)) \in$ $\{\mathbb{C}, \mathbb{R},[0, \infty)\}$ and continuity of $M$ implies continuity of $\left.M\right|_{f(X)}$. In the case when $f(X)=M(f(X)) \in\{\mathbb{R},[0, \infty)\}$ either

$$
M(y)=|y|^{c} \operatorname{sgn} y \quad \text { for } y \in \mathbb{R}
$$

or

$$
M(y)=y^{c} \quad \text { for } y \in[0, \infty)
$$

with a certain $c>0$ (see for example [16, Theorem 13.1.6]). If $M: \mathbb{C} \rightarrow \mathbb{C}$, then we put $H=M$. Hence conditions (iii) and (iv) hold.

If $f$ and $M$ are given by condition (i) or (ii) of this theorem, then it is easy to check that $f$ and $M$ satisfy (1.2). So consider the case when $f$ and $M$ are given by (iii) or (iv). Then $(M \circ f)(x)=g(x)+1$ for each $x \in X$ and hence $M \circ f$ satisfies (1.3). Thus, by multiplicativity of $\left.M\right|_{f(X)}$,

$$
M(f(x+M(f(x)) y))=M(f(x)) M(f(y))=M(f(x) f(y)) \quad \text { for } x, y \in X .
$$


Consequently, according to injectivity of $\left.M\right|_{f(X)}$, the functions $f$ and $M$ fulfill (1.2), which completes the proof.

Corollary 4.3. Assume $(\mathrm{H})$ and let $f: X \rightarrow \mathbb{K}$ with $M: \mathbb{K} \rightarrow \mathbb{K}$ satisfy (1.2). If $f$ is $\mathfrak{M}$-measurable (that is, $f^{-1}(U) \in \mathfrak{M}$ for every open subset $U$ of $\left.X\right)$ and $M$ is continuous on $\mathbb{K}$, then $M \circ f$ is continuous or $F \in \mathcal{I}$.

Proof. Assume that $M \circ f \neq$ const and, for an indirect proof, suppose that $F \notin \mathcal{I}$. Since $F=f^{-1}(\mathbb{K} \backslash\{0\})$, we have that $F \in \mathfrak{M}$. Take

$$
B(c):=\left\{b \in \mathbb{K}:|b-c|<\left|\frac{c}{2}\right|\right\} \quad \text { for } c \in \mathbb{K} .
$$

Then there exists a sequence $\left(c_{m}\right)_{m \in \mathbb{N}} \subset \mathbb{K}$ such that

$$
\mathbb{K} \backslash\{0\} \subset \bigcup_{m \in \mathbb{N}} B\left(c_{m}\right) .
$$

Hence

$$
F \subset \bigcup_{m \in \mathbb{N}} f^{-1}\left(B\left(c_{m}\right)\right)
$$

and thus there exists an $m \in \mathbb{N}$ such that the set $D=f^{-1}\left(B\left(c_{m}\right)\right) \in \mathfrak{M} \backslash \mathcal{I}$ and $|f(D)| \subset\left(0,2\left|c_{m}\right|\right)$. Moreover, if $f(X) \backslash\{0\} \subset S$, then $\operatorname{int}(S \backslash f(D)) \neq \emptyset$ (otherwise we would have $S \subset B\left(c_{m}\right)$ ) for an $m \in \mathbb{N}$, which contradicts $\{-1,1\} \not \subset B\left(c_{m}\right)$. Now, to end the proof it suffices to apply Theorem 4.1.

REMARK 4.4. Let $f: X \rightarrow \mathbb{K}$ and $M=\mathrm{id}_{\mathbb{K}}$ satisfy the assumptions of Theorem 4.1. Define a binary operation $\star: X^{2} \rightarrow X$ by $x \star y:=x+f(x) y$. Then it is easy to check that $(X, \star)$ is a semigroup if and only if $f$ satisfies the Gołąb-Schinzel equation (1.3). Consequently, in view of Theorem $4.1,(X, \star)$ is a semigroup if and only if $f$ is given by

$$
f(x)=g(x)+1 \quad \text { for } x \in X
$$

or

$$
f(x)=\max \{0, g(x)+1\} \quad \text { for } x \in X \text {. }
$$

\section{References}

[1] J. Aczél and S. Gołąb, 'Remarks on one-parameter subsemigroups of the affine group and their homo- and isomorphism', Aequationes Math. 4 (1970), 1-10.

[2] J. A. Baker, 'On some mathematical characters', Glas. Mat. 25(45) (1990), 319-328.

[3] K. Baron and Pl. Kannappan, 'On the Cauchy difference', Aequationes Math. 46 (1993), 112-118.

[4] A. Beck, H. H. Corson and A. B. Simon, 'The interior points of the product of two subsets of a locally compact group', Proc. Amer. Math. Soc. 9 (1958), 648-652.

[5] J. Brzdȩk, 'Subgroups of the group $Z_{n}$ and a generalization of the Gołąb-Schinzel functional equation', Aequationes Math. 43 (1992), 59-71. 
[6] J. Brzdęk, 'The Christensen measurable solutions of a generalization of the Gołąb-Schinzel functional equation', Ann. Polon. Math. 46(3) (1996), 195-205.

[7] J. Brzdęk, 'Bounded solutions of the Gołąb-Schinzel equation', Aequationes Math. 59 (2000), $248-254$.

[8] J. Brzdęk, 'The Gołąb-Schinzel equation and its generalizations', Aequationes Math. 70 (2005), $14-24$.

[9] J. Brzdęk, 'Generalizations of some results concerning microperiodic mappings', Manuscripta Math. 121 (2006), 265-276.

[10] J. Brzdęk, 'Continuity of measurable homomorphisms', Bull. Aust. Math. Soc. 78 (2008), $171-176$.

[11] S. Gołąb and A. Schinzel, 'Sur l'équation fonctionnelle $f(x+f(x) y)=f(x) f(y)$ ', Publ. Math. Debrecen 6 (1959), 113-125.

[12] E. Jabłońska, 'On solutions of a generalization of the Gołąb-Schinzel equation', Aequationes Math. 71 (2006), 269-279.

[13] E. Jabłońska, 'Continuity of Lebesgue measurable solutions of a generalized Gołąb-Schinzel equation', Demonstratio Math. 39(1) (2006), 91-96.

[14] E. Jabłońska, 'Functions having the Darboux property and satisfying some functional equation', Colloq. Math. 114(1) (2009), 113-118.

[15] Z. Kominek and M. Kuczma, 'Theorem of Bernstein-Doetsch, Piccard and Mehdi and semilinear topology', Arch. Math. (Basel) 52 (1989), 595-602.

[16] M. Kuczma, 'An introduction to the theory of functional equations and inequalities. Cauchy's equation and Jensen's inequality', PWN-Uniwersytet Śląski, Warszawa-Kraków-Katowice, 1985.

[17] J. C. Morgan II, 'Baire category from an abstract viewpoint', Fundamenta Math. 94 (1977), $13-23$.

[18] A. Mureńko, 'On solutions of a common generalization of the Gołąb-Schinzel equation and of the addition formulae', J. Math. Anal. Appl. 341 (2008), 1236-1240.

[19] J. C. Oxtoby, Measure and Category (Springer, New York, 1971).

[20] C. G. Popa, 'Sur l'équation fonctionnelle $f(x+f(x) y)=f(x) f(y)$ ', Ann. Polon. Math. 17 (1965), 193-198.

[21] W. Sander, 'Ein Beitrag zur Baire-Kategorie-Theorie', Manuscripta Math. 34 (1981), 71-83.

[22] W. Sierpiński, 'Sur la dualité entre la première catégorie et la mesure nulle', Fund. Math. 22 (1934), 276-280.

ELIZA JABŁOŃSKA, Department of Mathematics, Rzeszów University of Technology, W. Pola 2, 35-959 Rzeszów, Poland

e-mail: elizapie@prz.edu.pl 